\title{
Bottom-quark Fusion Processes at the LHC for Probing Z' Models and B-meson Decay Anomalies
}

\author{
Mohammad Abdullah, Mykhailo Dalchenko*, Bhaskar Dutta, Ricardo Eusebi, Peisi \\ Huang, Teruki Kamon, Denis Rathjens, Adrian Thompson \\ Texas A\&M University \\ E-mail: mykhailo.dalchenkodcern.ch
}

\begin{abstract}
Anomalies in B-meson decays reported by the LHC experiment suggest a violation of lepton universality. This could be explained by introducing a heavy neutral gauge boson Z' that selectively couples to third generation quarks and second generation leptons. While the performance of experimental searches for such models is good for large Z' masses, the low-mass region sensitivity is aversely affected by large SM background (mostly Drell-Yan).

In this study, we present a novel approach searching for a $Z$ ' decaying to muons in association with at least two jets at least one of which is bottom-tagged. We demonstrate that regions of model parameter space can be probed that current inclusive analyses are insensitive to.
\end{abstract}

ICHEP 2018, International Conference on High Energy Physics

4-11 July 2018

Seoul, Republic of Korea

${ }^{*}$ Speaker. 


\section{Introduction}

Combining $R_{K}$ and $R_{K^{*}}$ anomalies reported by LHCb and Belle collaborations ([1, 2, 3]), the overall deviation from the SM expectation is at least at a level of $4 \sigma[4,5]$. A massive $Z^{\prime}$ with a flavor changing $b s$ coupling, and a nonuniversal coupling to leptons could easily accommodate it. Current $Z^{\prime}$ searches assume dominant coupling to the first generation quarks and leptons, while the constraints on the $Z^{\prime}$ from flavor physics require the couplings to second and third generation fermions to be dominant.

We investigate a model with dominant couplings to $b$ quarks leading to bottom fermion fusion (BFF) production of the $Z^{\prime}$ with subsequent dimuon decay. The presence of additional $b$ jets in the dimuon final state helps to probe smaller $Z^{\prime}$ masses than the inclusive dimuon searches. This strategy can be applied to any model where $Z^{\prime}$ couples to $b$ quarks irrespective of solutions to $B$ anomalies.

Let's extend the SM by adding an extra $U(1)$ gauge group, which introduces a new gauge boson $Z^{\prime}$. With a flavor changing quark coupling and a nonuniversal lepton coupling, it can generate a contribution to the desired effective operator. In the lepton sector, the $Z^{\prime}$ needs to couple only to the muons. In order to preserve $S U(2)$ invariance, the $Z^{\prime}$ also couples to tops and muon neutrinos. Summarizing all the considerations above, we end up with the following lagrangian:

$$
\left.\mathscr{L} \supset Z^{\prime \mu}\left[g_{\mu} \bar{\mu} \gamma^{\mu} \mu+g_{\mu} \overline{v_{\mu}} \gamma^{\mu} P_{L} v_{\mu}+g_{b} \sum_{q=t, b} \bar{q} \gamma^{\mu} P_{L} q+\left(g_{b} \delta_{b s} \bar{s} \gamma^{\mu} P_{L} b+\text { h.c. }\right)\right)\right]
$$

\section{Collider Probes}

$B$ anomalies require upper bound on the $Z^{\prime}$ mass to be less than 5.5 (10) TeV in the 1 (2) sigma range, however it can be as light as $100 \mathrm{GeV}$ [6]. Within our model, the $Z^{\prime}$ is associated either with two $b$-jets (both $b$ quarks from gluon splitting), one $b$-jet (one $b$ quark from each of gluon splitting and sea quarks), or no $b$-jet (both $b$ quarks from sea quarks). Its possible decay modes are pairs of $b$ quarks, muons, muon neutrinos, and, if kinematically allowed, top quarks. In this study we focus on dimuon resonances.

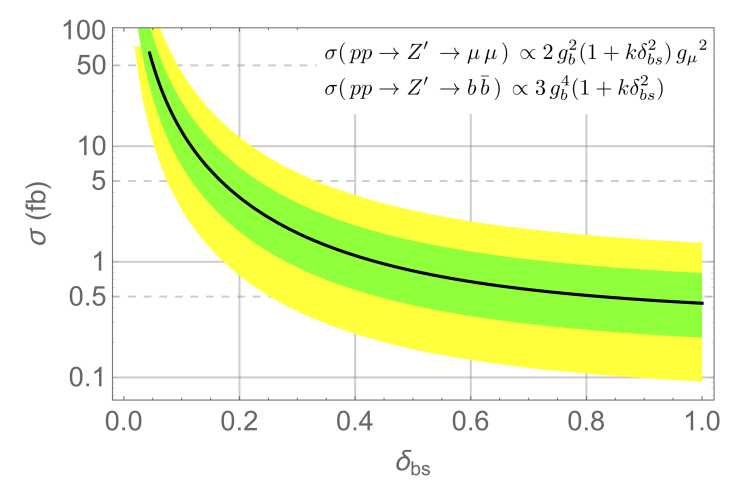

Figure 1: Production cross section for a BFF dimuon resonance as a function of $\delta_{b s}$ for a $350 \mathrm{GeV} Z^{\prime}$ and $g_{\mu}=0.13$. The central fit (black line) and the $1 \sigma$ (green shaded region) and $2 \sigma$ (yellow shaded region) contours of the $B$ anomalies are shown. 
Fig. 1 shows the production cross section for dimuon $+1(2) b$ final states and its dependency on couplings, where $k$ contains the $s$-quark PDF effect. For small $\delta_{b s}$ the flavor conserving contribution dominates the production of $Z^{\prime}$. When $\delta_{b s}$ is large but still satisfies the $B$ anomalies (so smaller $g_{b}$ ) the flavor violating contribution dominates.

Existing constraints are weak for $m_{Z^{\prime}} \leq 500 \mathrm{GeV}$ due to the large SM background contributions (Drell-Yan and $t \bar{t}$ ) in that region. We require two opposite sign muons and at least two jets with $p_{T}>30 \mathrm{GeV}$ with at least one of them passing a $b$-tag requirement, which reduces Drell-Yan $(+0,1,2$ jets) process contributions to the search region by a factor of $\mathscr{O}(100)$.

The remaining background is the dileptonic $t \bar{t}$ process that we suppress by further set of requirements:

- Top mass bound $M_{\mu b}$ : Of the two possible muon-jet parings, we choose the one with the smallest mass difference and require the heavier mass to be greater than $170 \mathrm{GeV}$.

- Leptonic versus hadronic activity: The scalar sum of transverse momenta of the leading OS muon pair $\left(L_{T}\right)$ must be larger than the scalar sum of transverse momenta of the leading bottom-tagged pair or bottom and non-tagged jet pair $\left(H_{\mathrm{T}}\right)$.

- Normalized missing transverse energy $\left(E_{\mathrm{T}}^{\mathrm{miss}}\right)$ : The ratio of $E_{\mathrm{T}}^{\text {miss }}$ to dimuon mass $\left(M\left(\mu^{+} \mu^{-}\right)\right)$ is restricted to below 0.2 to reject events with real sources of $E_{\mathrm{T}}^{\text {miss }}$.

\begin{tabular}{|l|r|r|r|r|}
\hline & preselection & $M_{\mu b}$ & $H_{\mathrm{T}}-L_{\mathrm{T}}$ & $E_{\mathrm{T}}^{\mathrm{miss}} / M\left(\mu^{+} \mu^{-}\right)$ \\
\hline$t \bar{t}$ & $8 \%$ & $17 \%$ & $26 \%$ & $27 \%$ \\
\hline $\mathrm{SM} Z$ & $0.2 \%$ & $41 \%$ & $32 \%$ & $54 \%$ \\
\hline$Z^{\prime} 200$ & $7 \%$ & $60 \%$ & $74 \%$ & $89 \%$ \\
\hline$Z^{\prime} 350$ & $10 \%$ & $82 \%$ & $90 \%$ & $97 \%$ \\
\hline
\end{tabular}

Table 1: Efficiency of selection requirements for three different mass points assuming $\delta_{b s}=0$ with a dimuon $t \bar{t}$ background. Listed requirements are to be read as accumulative from left to right with each efficiency denoting that additional requirement's efficiency after applying all other selections in columns to its left.

Table 1 contains the efficiencies of the aforementioned selection requirements on dileptonic $t \bar{t}$, SM $Z$ and two different mass scenarios for the $Z^{\prime}$ model.

\section{Results}

We fit the dimuon mass spectrum dependence upon $\delta_{b s}$ with a linear fit for each mass point by generating several differently $\delta_{b s}$-valued samples with constant $g_{b}$. Then, we fit the resulting absolute values of slopes and intercepts versus $Z^{\prime}$ mass with a logarithmic fit each to determine a function describing the signal acceptance $A$ over the complete parameter space:

$$
A\left(m_{Z^{\prime}}, \delta_{b s}\right)=\left(0.063-0.026 \delta_{b s}\right) \ln \left(\frac{m_{Z^{\prime}}}{\mathrm{GeV}}\right)-0.268+0.11 \delta_{b s}
$$

We use $g_{\mu} \sim 1$ to calculate the $Z^{\prime}$ decay width to make sure our bound is valid for such high values of couplings. The values dictated by the $B$ anomalies are much smaller and would lead to a narrower width and hence a larger significance. 

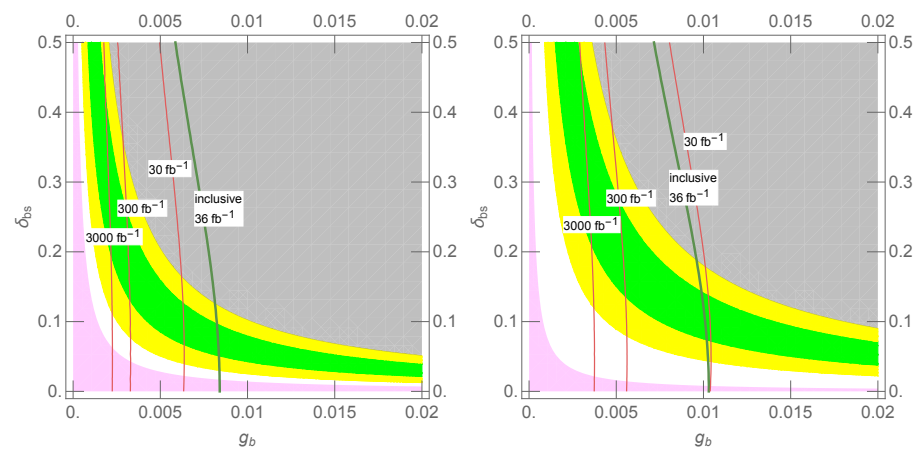

Figure 2: The current and future expected LHC limits for various luminosities for 200 (left) and $350 \mathrm{GeV}$ (right) $Z^{\prime}$ masses. Green lines refer to the current reach of an inclusive dimuon search while red lines show the expected power of a 2(1) $b+$ dimuon search. The yellow and green shaded regions correspond to $2 \sigma$ and $1 \sigma$ bands of the best fit to the $B$ anomalies for the chosen values of $g_{\mu}(0.08$, and 0.14 respectively). The grey shaded area is ruled out by the $B_{s}-\bar{B}_{s}$ mixing constraint. In the pink region, the required $g_{\mu}$ to fit the $B$ anomalies is ruled out by neutrino trident production.

We show the LHC projected reach for 200 , and $350 \mathrm{GeV} Z^{\prime}$ masses in the $\delta_{b s}-g_{b}$ parameter space in Fig. 2 along with constraints from $B_{s}-\bar{B}_{s}$ mixing [6] and trident production [7].

For relatively low $m_{Z^{\prime}}$, a 2(1)b + dimuon search as proposed in this work is more efficient in mitigating the background than the inclusive dimuon analogue leading to enhanced limits. The prospects for testing the entire parameter space of such models for some $Z^{\prime}$ masses appear to be complimentary in the existing and upcoming LHC program.

\section{References}

[1] LHCB collaboration, R. Aaij et al., Measurement of Form-Factor-Independent Observables in the Decay $B^{0} \rightarrow K^{* 0} \mu^{+} \mu^{-}$, Phys. Rev. Lett. 111 (2013) 191801 [1308.1707].

[2] LHCB collaboration, R. Aaij et al., Angular analysis of the $B^{0} \rightarrow K^{* 0} \mu^{+} \mu^{-}$decay using $3 f^{-1}$ of integrated luminosity, JHEP 02 (2016) 104 [1512.04442].

[3] Belle collaboration, A. Abdesselam et al., Angular analysis of $B^{0} \rightarrow K^{*}(892)^{0} \ell^{+} \ell^{-}$, in Proceedings, LHCSki 2016 - A First Discussion of 13 TeV Results: Obergurgl, Austria, April 10-15, 2016, 2016, 1604.04042 , http://inspirehep.net/record/1446979/files/arXiv:1604.04042.pdf.

[4] G. D’Amico, M. Nardecchia, P. Panci, F. Sannino, A. Strumia, R. Torre et al., Flavour anomalies after the $R_{K^{*}}$ measurement, JHEP 09 (2017) 010 [1704 . 05438].

[5] B. Capdevila, A. Crivellin, S. Descotes-Genon, J. Matias and J. Virto, Patterns of New Physics in $b \rightarrow s \ell^{+} \ell^{-}$transitions in the light of recent data, JHEP 01 (2018) 093 [1704.05340].

[6] W. Altmannshofer, S. Gori, M. Pospelov and I. Yavin, Quark flavor transitions in $L_{\mu}-L_{\tau}$ models, Phys. Rev. D89 (2014) 095033 [1403.1269].

[7] W. Altmannshofer, S. Gori, M. Pospelov and I. Yavin, Neutrino Trident Production: A Powerful Probe of New Physics with Neutrino Beams, Phys. Rev. Lett. 113 (2014) 091801 [1406.2332]. 